China Ocean Eng., 2021, Vol. 35, No. 6, P. 789-801

DOI: https://doi.org/10.1007/s13344-021-0070-8, ISSN 0890-5487

http://www.chinaoceanengin.cn/ E-mail: coe@nhri.cn

\title{
Fluid Resonance Between Twin Floating Barges with Roll Motion Under Wave Action
}

\author{
LI Shu, TENG Bin* \\ State Key Laboratory of Coastal and Offshore Engineering, Dalian University of Technology, Dalian 116024, China
}

Received December 27, 2020; revised August 27, 2021; accepted September 20, 2021

C2021 Chinese Ocean Engineering Society and Springer-Verlag GmbH Germany, part of Springer Nature

\begin{abstract}
The wave-induced fluid resonance between twin side-by-side rectangular barges coupled with the roll motion of the twin barges is investigated by both numerical simulation and physical model test. A 2D numerical wave flume, based on an open source computational fluid dynamics (CFD) package OpenFOAM, is applied for the numerical simulation. After numerical validations and convergent verifications, the characteristics of the fluid resonance in the gap between the twin rolling side-by-side barges are examined. The resonant frequency of the oscillating fluid in the gap between the twin rolling barges decreases compared with that between the twin fixed barges. Generally, the twin barges roll in the opposite directions, and their equilibrium positions lean oppositely with respect to the initial vertical direction. A physical model test is carried out for a further investigation, in which the twin barges are set oppositely leaning and fixed. From the present experimental results, a linear decrease of the resonant frequency with the increasing leaning angle is found. Combined with the numerical results, the deflection of the equilibrium positions of the twin barges is a relevant factor for the resonant frequency. Besides, the effects of the gap width and incident wave height on the fluid resonance coupled with roll motion are examined.
\end{abstract}

Key words: fluid resonance in a narrow gap, roll motion of the twin barges, OpenFOAM

Citation: Li, S., Teng, B., 2021. Fluid resonance between twin floating barges with roll motion under wave action. China Ocean Eng., 35(6): 789-801, doi: https://doi.org/10.1007/s13344-021-0070-8

\section{Introduction}

Floating structures in proximity are often found in the ocean engineering, for example, the offloading of liquefied gas from a floating production storage and offloading (FPSO) to an oil tank or liquefied natural gas (LNG) ship. The large amplitude fluid resonance can be observed in the narrow gap formed by the closely spaced structures when the incident wave frequency is close to the natural frequency of the fluid confined by the gap. As the floating structures are long enough and incident waves are normal to the floating structures, a 2D assumption can be made for the purpose of convenience. By further assuming that the floating structures are fixed, the wave-induced fluid resonance in the narrow gap has been extensively studied, including theoretical analyses, laboratory tests and numerical simulations. Miao et al. (2001) studied the wave interaction of twin floating barges with a small gap between them theoretically. It proved that the resonance takes place around $k L_{\mathrm{B}}=n \pi(n=1,2,3, \ldots, \infty)$, where $k$ is the incident wave number and $L_{\mathrm{B}}$ is the length of the barge. Fluid resonance between two floating horizontal circle cylinders is investigated analytically (McIver, 2005), so is the fluid resonance between a floating horizontal circle cylinder and a vertical wall (McIver and Porter, 2016).

Saitoh et al. (2006) conducted a 2D experiment to study the fluid resonance between two fixed barges. The experimental results indicated that the maximal amplitude of the resonant wave motion excited in the narrow gap approached up to five times the incident wave amplitude approximately. Iwata et al. (2007) focused on the case of three fixed rectangular modules with two gaps and investigated the effects of the relevant parameters on the resonant frequencies. Wang and Zou (2007) studied the wave force on the barge in front of a terminal with a relatively shallow water when fluid resonance took place. Tan et al. (2016) took the edge shapes of the twin barges into consideration for model experiments and found the characteristics of the resonant frequency and wave height with various edge shapes. Ning et al. (2018) investigated the effects of the various draughts for twin barges in proximate on the frequency and wave height in the gap when resonance happened.

Based on the linear potential flow theory, Choi and Hong (2002), Hong et al. (2005) and Kashiwagi et al. (2005) employed higher order boundary element method 
(HOBEM) to investigate the hydrodynamics of the FPSO and LNG moored tandem or side-by-side. Scaled boundary finite element method (SBFEM) was employed to study the scattering problem of twin barges in proximate ( $\mathrm{Li}$ et al., 2005). Sun et al. (2010, 2015) and Jin et al. (2017) studied the fluid resonance frequency in the gap of the side-by-side mooring FLNG and LNG and the effects of the second-order wave force in frequency domain and time domain, respectively. A fully nonlinear potential model in time domain was utilized to study the fluid resonance in a narrow gap (Yan et al., 2009). Though the resonant frequency can be estimated accurately, the potential models tend to overpredict the wave amplitudes in the narrow gap because the physical energy is dissipated due to fluid viscosity, vortex shedding and even turbulence cannot be taken into account. Therefore, CFD models are employed to investigate the wave-induced fluid resonance in the narrow gaps between fixed barges. Lu et al. $(2008,2010)$ investigated the gap resonance between two and three fixed identical barges, respectively. It was revealed that the wave energy was dissipated by the vortices in the gap when resonance happened. Furthermore, the dissipation due to the friction of the solid boundary is approximately $1 / 6$ of the mechanical energy (Lu et al., 2012). Jiang et al. (2018) considered different sizes of the two fixed barges to investigate the resonant frequency and wave force. Smoothed particles hydrodynamics (SPH) was employed to study the energy dissipation between a fixed barge and a vertical wall when resonance happened (Meringolo et al., 2018). Besides, constrained interpolation profile (CIP) was utilized to invesigate the wave force impacting on the floating barges when fluid resonance took place (Zhang et al., 2018). Tan et al. (2019) developed a viscous damping model for piston mode resonance based on the conservation of the energy. Though the fluid viscosity and vortex shedding can be taken into consideration, the interaction between the incident wave and the motion of the floating barge has not been considered. Kristiansen and Faltinsen (2010) studied the fluid resonance between a ship and a terminal by $2 \mathrm{D}$ physical experiments and numerical simulation. In the model the ship section was allowed to oscillate in three degrees of freedom: sway, heave and roll. The study indicates that large piston-mode motion occurs when the sway motion is towards the terminal and the heave motion is downwards the terminal simultaneously.

However, the fluid resonance in a narrow gap between twin rolling side-by-side floating barges received scarce attention. Therefore, the main purpose of this work is to examine the wave-induced coupling effects of the fluid resonance with the roll motion between twin side-by-side floating barges. Based on the present numerical results, a physical model test of the gap resonance between twin oppositely leaning and fixed side-by-side floating barges is carried out for a further investigation. Besides, the effects of relevant parameters for fluid resonance coupled with roll motion between twin side-by-side floating barges are focused on. The contents are organized as follows. The numerical model based on OpenFOAM in this work is described in Section 2. Numerical validations are presented in Section 3. The numerical results of coupling fluid resonance and roll motion of the twin floating barges are discussed in Section 4. The setup and results of the physical model test for the fluid resonance between twin oppositely leaning and fixed side-by-side floating barges are introduced in Section 5. In Section 6, the numerical results of fluid resonance coupled with roll motion between twin side-by-side floating barges with various parameters are illustrated. Finally, the conclusions of this work are drawn in Section 7.

\section{Numerical model}

The present numerical wave flume is based on the OpenFOAM package, in which the governing equations are Navier-Stokes equations. The interface between water phase and air phase is captured by the volume of fluid (VOF) method (Hirt and Nichols, 1981). Because the functional modules of the wave generation and wave absorption are absent in the OpenFOAM package, the momentum equation is modified in order to satisfy the requirements of the wave simulation. The roll motion of the floating barge about its fixed axis is computed by the angular momentum conservative equation. Fig. 1 shows the sketch of the present numerical model.

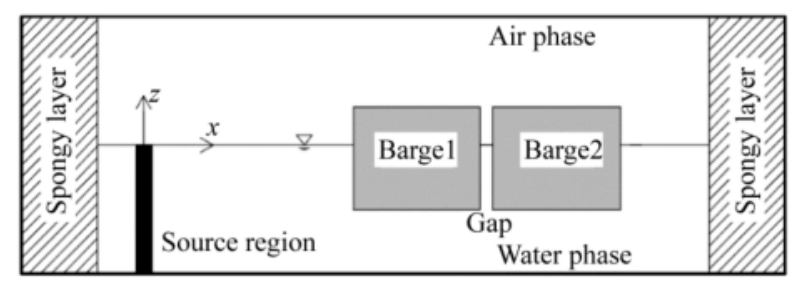

Fig. 1. Sketch of the present numerical model.

\subsection{Governing equations}

In terms of incompressible two-phase fluid, the NavierStokes equations can be written as follows based on the Arbitrary Lagrangian-Eulerian theory (Hirt et al., 1997),

$\nabla \cdot \boldsymbol{U}=0$

$$
\begin{gathered}
\frac{\partial(\rho \boldsymbol{U})}{\partial t}+\nabla \cdot\left[\rho\left(\boldsymbol{U}-\boldsymbol{U}^{m}\right) \boldsymbol{U}\right]=-\nabla p+\rho \boldsymbol{g}+ \\
\nabla \cdot(\mu \nabla \boldsymbol{U})-f_{\sigma}+\rho \boldsymbol{S}-\rho \Theta \boldsymbol{U},
\end{gathered}
$$

where $\rho$ is the fluid density, $\boldsymbol{U}$ is the fluid velocity, $p$ is the pressure, $\mu$ is the fluid dynamic viscosity, $\boldsymbol{f}_{\sigma}$ is the surface tension which only exists at the interface between the air phase and water phase, $S$ is the source function used to generate waves, $\Theta$ is the artificial damping term, $\boldsymbol{U}^{m}$ is the velocity of the moving mesh motion in order to deal with the fluid and structure interaction and $g$ is the acceleration due 
to gravity. The expressions of $f_{\sigma}, S$ and $\Theta$ will be introduced in the following text.

\subsection{Interface capturing approach}

The VOF method (Hirt and Nichols, 1981) is employed to capture the interface between the air phase and water phase. In the VOF method, each of the two phases is considered to have a separately defined volume fraction. When the cell is empty of water but filled with air, the value of volume fraction function is 0 . By the contrary, when the cell is full of water without air, the value of volume fraction function is 1 . When the interface cuts the cell, the function is between 0 and 1 . That is

$\rho=\varphi \rho_{\mathrm{w}}+(1-\varphi) \rho_{\mathrm{a}}, \quad \mu=\varphi \mu_{\mathrm{w}}+(1-\varphi) \mu_{\mathrm{a}}$;

$\varphi= \begin{cases}\varphi=0, & \text { air } \\ 0<\varphi<1, & \text { interface } \\ \varphi=1, & \text { water }\end{cases}$

where $\varphi$ is the volume fraction function, $\rho_{\mathrm{w}}$ and $\rho_{\mathrm{a}}$ are the densities of water and air, respectively, and $\mu_{\mathrm{w}}$ and $\mu_{\mathrm{a}}$ are the dynamic viscosities of water and air, respectively.

The volume fraction function is determined by solving an advection equation

$\frac{\partial \varphi}{\partial t}+\nabla \cdot\left[\left(\boldsymbol{U}-\boldsymbol{U}^{m}\right) \varphi\right]+\nabla \cdot\left[\boldsymbol{U}_{\mathrm{r}} \varphi(1-\varphi)\right]=0$,

where $\boldsymbol{U}=\varphi \boldsymbol{U}_{\mathrm{w}}+(1-\varphi) \boldsymbol{U}_{\mathrm{a}}$ is applied to describe the fluid velocity of a two-phase fluid, and $\boldsymbol{U}_{\mathrm{r}}=\boldsymbol{U}_{\mathrm{w}}-\boldsymbol{U}_{\mathrm{a}}$ is the relative velocity (Rusche, 2002). $\boldsymbol{U}_{\mathrm{w}}$ and $\boldsymbol{U}_{\mathrm{a}}$ are the velocities of water and air, respectively.

\subsection{Surface tension}

For a two-phase fluid flow, surface tension exists at the interface between the air and water, which is normal to the interface. That is,

$\boldsymbol{f}_{\sigma}=\sigma \kappa \nabla \varphi$

where $\sigma$ is the constant of the surface tensor, which is generally equal to 0.074 (Ubbink and Issa, 1999), and $\kappa$ is the interface curvature, which can be computed by

$\kappa=\nabla \cdot \boldsymbol{n}$,

in which $\boldsymbol{n}$ is the unit outward normal vector:

$\boldsymbol{n}=\frac{\nabla \varphi}{|\nabla \varphi|}$.

\subsection{Wave generation}

A source function is added to the momentum equation to generate waves (Wei et al., 1999). In the Cartesian coordinate system, the expression of the momentum source function is

$$
\begin{aligned}
& S_{x}=g(2 \beta x) \mathrm{e}^{-\beta\left(x-x_{s}\right)^{2}} \frac{1}{4 \pi^{2}} \int_{-\infty}^{+\infty} \int_{-\infty}^{+\infty} \frac{\mathrm{i} D\left(k_{y}, \omega\right)}{\omega} \mathrm{e}^{\mathrm{i}\left(k_{y} y-\omega t\right)} \mathrm{d} \omega \mathrm{d} k_{y} ; \\
& S_{y}=g \mathrm{e}^{-\beta\left(x-x_{s}\right)^{2}} \frac{1}{4 \pi^{2}} \int_{-\infty}^{+\infty} \int_{-\infty}^{+\infty} \frac{k_{y} D\left(k_{y}, \omega\right)}{\omega} \mathrm{e}^{\mathrm{i}\left(k_{y} y-\omega t\right)} \mathrm{d} \omega \mathrm{d} k_{y}
\end{aligned}
$$

$S_{z}=0$

where $\alpha=\frac{z_{\alpha}}{h}\left(\frac{z_{\alpha}}{2 h}+1\right), \quad \alpha_{1}=\alpha+1 / 3$ and $z_{\alpha}=-0.530 h(h$ is the water depth) are the parameters (Nwogu, 1993); $D=\frac{H\left(\omega^{2}-\alpha_{1} g k^{4} h^{3}\right) \cos \theta}{\omega I_{1} k\left[1-\alpha(k h)^{2}\right]}, \quad I_{1}=\sqrt{\frac{\pi}{\beta}} \mathrm{e}^{-k^{2} /(4 \beta)}$ and $\beta=80 /$ $(\delta L)^{2}$ are the parameters related to the incident wave (Wei et al., 1999). Furthermore, $\delta=2 W / L$ is a control parameter for the source width $W, L$ is the wavelength, $k_{y}=k \sin \theta$ is the wave number in $y$ direction, $\theta$ is the angle between the propagation direction and $x$-axis, $x_{\mathrm{s}}$ is the center of source region, $\omega$ is the angular frequency of the incident wave, $h$ is the water depth, and $H$ is the incident wave height.

\subsection{Wave damping zone}

In this work, the damping zone is applied to absorb waves at the outlet boundary. The expression of the artificial damping term is

$\Theta= \begin{cases}0, & 0<x<x_{0} \\ \frac{\left(x-x_{0}\right)}{\left(x_{1}-x_{0}\right)} \Theta_{1}, & x_{0}<x<x_{1}\end{cases}$

where $x_{0}$ is the start position of the damping zone, $x_{1}$ is the end position and $\Theta_{1}$ is the damping coefficient which can be determined empirically (Larsen and Darcy, 1983; Romate, 1992).

\subsection{Roll motion of a rigid body}

Each of the twin barges is fixed along an axis and has only one degree of freedom of motion, namely, rotation. The roll motion of a floating barge can be determined by the rotation equation, derived from the conservation of the rotation moment as:

$I \ddot{\zeta}=M_{\mathrm{W}}+M_{\mathrm{G}}$,

where $I$ is the moment of inertia, $\zeta$ is angular displacement of the rotating body, $M_{\mathrm{W}}$ is the torque produced by the fluid which includes pressure torque and viscous torque, and $M_{\mathrm{G}}$ is the torque due to gravity. In this work, the roll direction along the clockwise is defined as positive.

\subsection{Moving mesh technique}

The moving mesh technique is applied for modeling the movement of the roll motion of a rigid body subjected to incident waves. The topology of the mesh does not change, but only cell shapes deform. The positions of the mesh points in the field are determined by solving a Laplace equation. That is

$$
\nabla \cdot\left(\gamma \nabla \boldsymbol{U}^{m}\right)=0
$$

where $\gamma$ is a diffusivity parameter, which is chosen as the inverse of square of the distance to the roll body boundary (Jasak and Tukovic, 2007). The new positions of the mesh points can be obtained as:

$\boldsymbol{x}_{\text {new }}=\boldsymbol{x}_{\text {old }}+\Delta t \cdot \boldsymbol{U}^{m}$, 
new position, and $\Delta t$ is the time step.

An example of the moving mesh is shown in Fig. 2, which is employed to address the roll motion of the twin barges. Multi-block grid system is utilized around the barges.

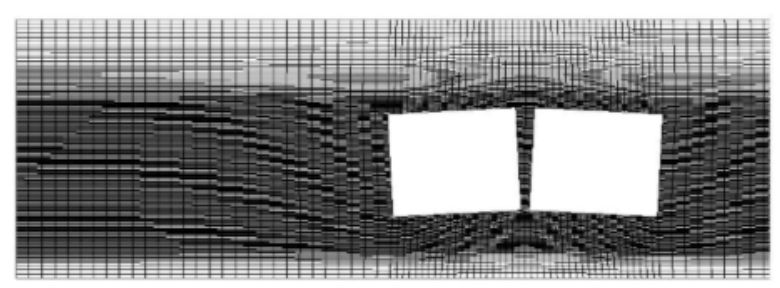

Fig. 2. An example for the moving mesh.

\section{Numerical validation}

The validation of the present numerical model includes three aspects: the generation and propagation of water waves, wave-induced gap resonance between twin fixed rectangular barges, and the free roll decay motion of a rectangular barge.

\subsection{Linear wave generation and propagation over flat bed}

A 2D wave flume is set up as shown in Fig. 3. The length of the wave flume is $30 \mathrm{~m}$ and the water depth is $h=0.5 \mathrm{~m}$. The middle of the source region locates at $x_{\mathrm{s}}=0$ and damping layers begin at $x=-3 \mathrm{~m}$ and $x=17 \mathrm{~m}$ in the two ends of the wave flume, respectively. The length of the source region $W$ is equal to the wavelength $L$.

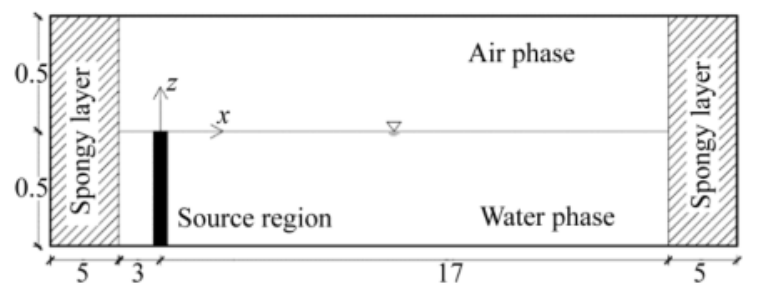

Fig. 3. Sketch of the numerical flume (unit: $m$ )

For a linear monochromatic wave, the momentum source function can be simplified as:

$S_{x}=-g(2 \beta x) \exp \left[-\beta\left(x-x_{\mathrm{s}}\right)^{2}\right] \frac{D}{\omega} \sin \left(k_{y} y-\omega t\right) ;$

$S_{y}=g \exp \left[-\beta\left(x-x_{\mathrm{s}}\right)^{2}\right] \frac{k_{y} D}{\omega} \cos \left(k_{y} y-\omega t\right)$;

$S_{z}=0$.

Under the condition of two-dimension, the momentum source function can be further simplified, namely $\theta=0$. That is

$S_{x}=-g(2 \beta x) \exp \left[-\beta\left(x-x_{\mathrm{s}}\right)^{2}\right] \frac{D}{\omega} \sin (-\omega t) ;$

$S_{y}=0$;

$S_{z}=0$.
The parameters of the incident wave for the numerical validation are the wave height $H_{0}=0.024 \mathrm{~m}$ and the wave period $T=1.189 \mathrm{~s}$. Three meshes, with cell sizes of 50 $\mathrm{mm} \times 3.75 \mathrm{~mm}$ (coarse mesh), $25 \mathrm{~mm} \times 2 \mathrm{~mm}$ (medium mesh) and $12.5 \mathrm{~mm} \times 1 \mathrm{~mm}$ (fine mesh), are adopted to examine the convergence on the cell size. The time series of the wave profile measured by a numerical wave gauge $11.975 \mathrm{~m}$ away from the center of the source region is shown in Fig. 4, which indicates that the result for a medium cell size of $25 \mathrm{~mm} \times 2 \mathrm{~mm}$ has converged.

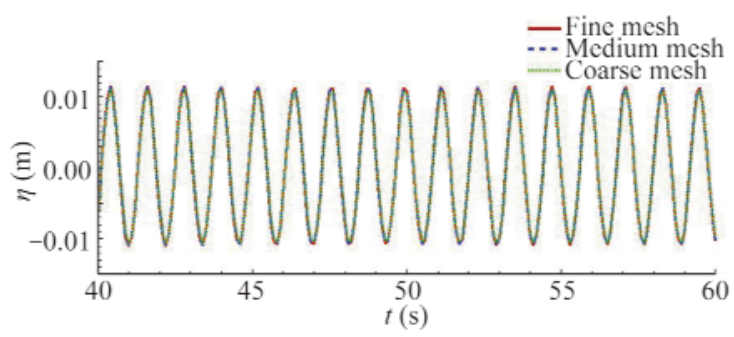

Fig. 4. Comparison of wave heights among different meshes.

The Airy wave theory is employed for comparison, corresponding to the mentioned method for wave generation. Fig. 5 shows the wave profile of the present numerical results at $t=30 \mathrm{~s}$ compared with the theoretical solution, in which $A_{0}$ is the wave amplitude. The errors of the wave amplitude and frequency in the region where the structures will be arranged $(x / L=4-8)$ are approximately $5.0 \%$ and $0.50 \%$, respectively, with the Fourier analysis being employed. Good agreements are obtained regardless of the numerical results in the source region, which indicates that the present numerical wave flume can correctly simulate the propagation of Airy wave.

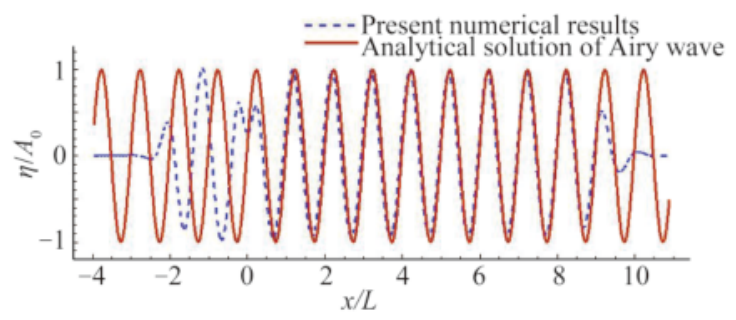

Fig. 5. Comparison between the present numerical results and analytical solution.

\subsection{Wave-induced gap resonance of twin fixed rectangular barges}

The wave-induced fluid resonance in the narrow gap between twin fixed rectangular barges is employed for the numerical validation. The numerical setup is corresponding to the physical experiments (Saitoh et al., 2006), which is shown in Fig. 6. The parameters of the twin rectangular barges are given in Table 1 . The water depth $h=0.5 \mathrm{~m}$ and incident wave height $H_{0}=0.024 \mathrm{~m}$ are kept as constants. The various periods of incident waves are shown in Table 2. 


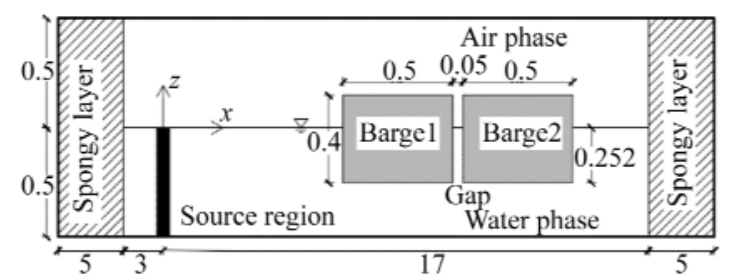

Fig. 6. Sketch of numerical setup in accordance with available experiments (unit: $\mathrm{m}$ ).

Table 1 Parameters of twin rectangular barges

\begin{tabular}{llll}
\hline $\begin{array}{l}\text { Width of } \\
\text { barges } B_{x}\end{array}$ & $\begin{array}{l}\text { Height of } \\
\text { barges } B_{z}\end{array}$ & $\begin{array}{l}\text { Draft of } \\
\text { barges } D_{\mathrm{r}}\end{array}$ & $\begin{array}{l}\text { Gap width } \\
B_{\mathrm{g}}\end{array}$ \\
\hline $0.5 \mathrm{~m}$ & $0.4 \mathrm{~m}$ & $0.252 \mathrm{~m}$ & $0.05 \mathrm{~m}$ \\
\hline
\end{tabular}

Table 2 Periods of incident waves

\begin{tabular}{lll}
\hline Case No. & $T(\mathrm{~s})$ & $k h$ \\
\hline 1 & 1.000 & 2.078 \\
2 & 1.070 & 1.848 \\
3 & 1.111 & 1.736 \\
4 & 1.123 & 1.705 \\
5 & 1.135 & 1.674 \\
6 & 1.149 & 1.643 \\
7 & 1.176 & 1.584 \\
8 & 1.189 & 1.156 \\
9 & 1.211 & 1.512 \\
10 & 1.222 & 1.491 \\
11 & 1.234 & 1.469 \\
12 & 1.248 & 1.444 \\
13 & 1.276 & 1.396 \\
14 & 1.299 & 1.360 \\
15 & 1.319 & 1.330 \\
16 & 1.334 & 1.309 \\
17 & 1.372 & 1.257 \\
18 & 1.430 & 1.186 \\
\hline
\end{tabular}

The variation of the non-dimensional averaging wave amplitude $H_{\mathrm{g}} / H_{0}$ in the gap with respect to non-dimensional wave number $k h$ is plotted in Fig. 7. The experiment data (Saitoh et al., 2006) are also included for comparison. It shows that the present numerical results agree well with the

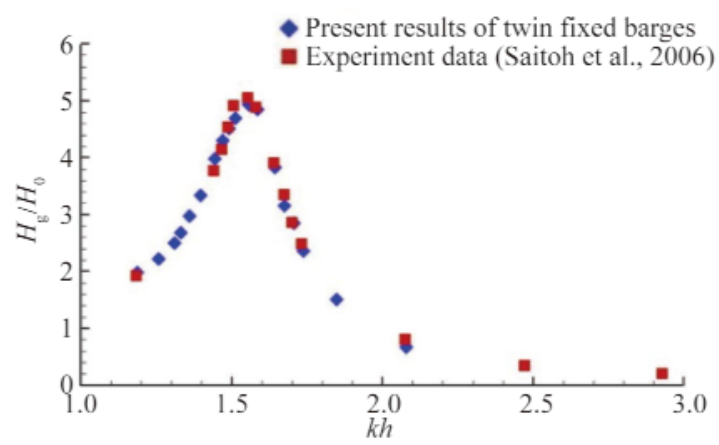

Fig. 7. Comparison of wave amplitudes in the gap between twin fixed barges. available experimental data. The period for the maximum wave height in the gap between twin fixed barges is $T=1.189 \mathrm{~s}$.

\subsection{Free roll decay of a rectangular barge}

To further validate the fluid structure interaction algorithm, the free roll decay of a rectangular barge is simulated. In accordance with the numerical simulation (Calderer et al., 2014) and the physical experiments (Jung et al., 2006), the parameters are set as follows. The width and height of the barge are $0.3 \mathrm{~m}$ and $0.2 \mathrm{~m}$, respectively. The water depth is $0.9 \mathrm{~m}$ and the draft of the barge is $0.05 \mathrm{~m}$. The barge rotates with respect to its center of gravity, which is located at the still water level. The rotational inertia of the barge is $I=0.236 \mathrm{~kg} \cdot \mathrm{m}^{2}$. The initial deflection is $15^{\circ}$ with respect to the still water level. The configuration is illustrated in Fig. 8. The time series of the roll displacement are compared with the available numerical results (Calderer et al., 2014) and experimental data (Jung et al., 2006) in Fig. 9, which illustrates that the present numerical model works well.

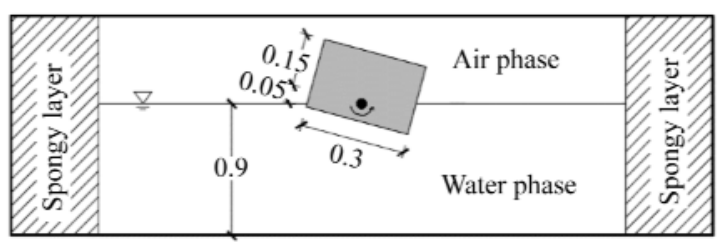

Fig. 8. Sketch of numerical setup for free roll decay of a rectangular barge.

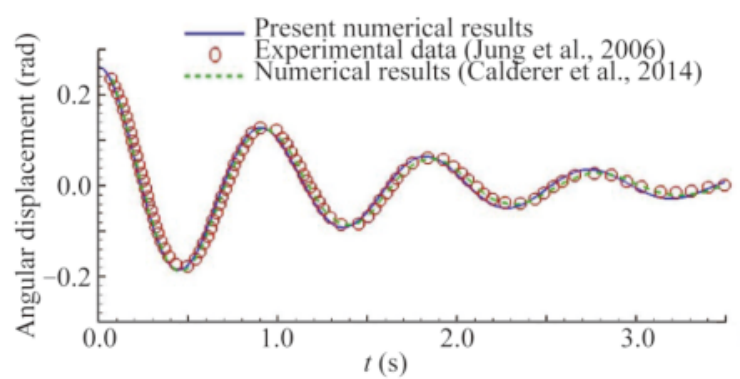

Fig. 9. Comparison of roll displacement between present numerical results and available data.

\section{Numerical results of coupling fluid resonance with roll motion of twin floating barges}

By employing the present numerical model, the fluid resonance coupled with roll motion in the gap between twin side-by-side barges is simulated. The wave height in the gap and the characteristics of the roll motion for the twin barges are focused on with various periods of incident waves.

\subsection{Numerical setup}

The numerical setup and computational parameters are 
shown in Fig. 10, which are identical to those in Section 3.2, except that each barge has a roll degree of freedom. The barge draught and gap width are $D_{\mathrm{r}}=0.252 \mathrm{~m}$ and $B_{\mathrm{g}}=0.05 \mathrm{~m}$ in this numerical simulation, respectively. The initial state of the barges is set under the hydrostatic equilibrium condition. The mass and roll moment of inertia of each barge are $m=126 \mathrm{~kg} / \mathrm{m}$ and $I=4.305 \mathrm{~kg} \cdot \mathrm{m}^{2} / \mathrm{m}$, respectively. The rotation center of each barge is located at the corresponding geometry center. The water depth and incident wave height remain as $h=0.5 \mathrm{~m}$ and $H_{0}=0.024 \mathrm{~m}$, respectively. Table 3 shows various periods of the incident waves.

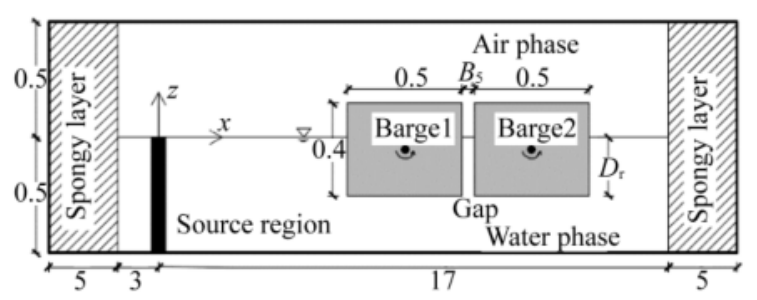

Fig. 10. Sketch of coupling fluid resonance and roll motion in the narrow gap (unit: $\mathrm{m}$ ).

Table 3 Parameters of incident waves

\begin{tabular}{|c|c|c|}
\hline Case No. & $T(\mathrm{~s})$ & $k h$ \\
\hline 1 & 1.000 & 2.078 \\
\hline 2 & 1.070 & 1.848 \\
\hline 3 & 1.111 & 1.736 \\
\hline 4 & 1.123 & 1.705 \\
\hline 5 & 1.136 & 1.674 \\
\hline 6 & 1.149 & 1.643 \\
\hline 7 & 1.176 & 1.584 \\
\hline 8 & 1.189 & 1.156 \\
\hline 9 & 1.211 & 1.512 \\
\hline 10 & 1.222 & 1.491 \\
\hline 11 & 1.234 & 1.469 \\
\hline 12 & 1.248 & 1.444 \\
\hline 13 & 1.276 & 1.396 \\
\hline 14 & 1.277 & 1.395 \\
\hline 15 & 1.280 & 1.390 \\
\hline 16 & 1.283 & 1.385 \\
\hline 17 & 1.287 & 1.380 \\
\hline 18 & 1.290 & 1.375 \\
\hline 19 & 1.293 & 1.370 \\
\hline 20 & 1.296 & 1.365 \\
\hline 21 & 1.299 & 1.360 \\
\hline 22 & 1.319 & 1.330 \\
\hline 23 & 1.334 & 1.309 \\
\hline 24 & 1.372 & 1.257 \\
\hline 25 & 1.430 & 1.186 \\
\hline
\end{tabular}

4.2 Resonant frequency predicted by the potential theory

Before the numerical simulation, the resonant frequency of a single barge in the roll direction employed for the numerical investigation needs to be known. The potential model can predict the resonant frequency both accurately and conveniently. Therefore, a linear potential model in frequency domain based on HOBEM (Teng and Taylor, 1995) is employed for the prediction of the resonant frequency. Fig. 11 shows the potential results of the roll amplitudes for a single barge, including that for the aforementioned twin barges in Section 4.1. The resonant frequency of a single barge is $k h=2.57(T=0.890 \mathrm{~s})$, which is higher than that of the twin barges $k h=1.57$ ( $T=1.182 \mathrm{~s})$. No relatively violent roll motion of the twin barges is found at the resonant frequency of the single barge, so the fluid oscillation should be relatively mild at that frequency. It means that the oscillating fluid in the gap mainly governs the roll motion of the twin barges.

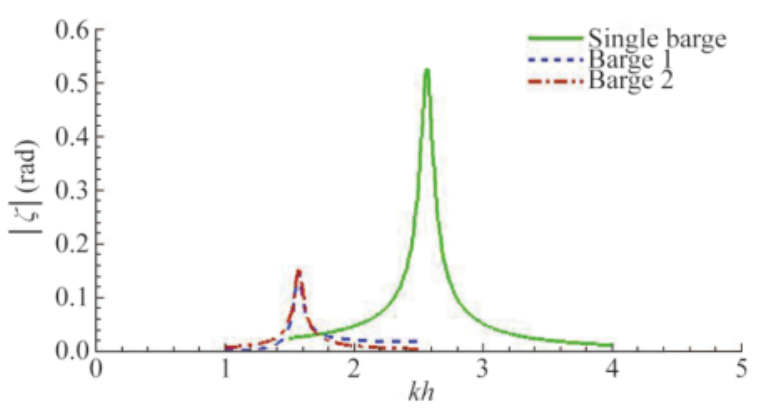

Fig. 11. Roll amplitudes predicted by the potential model.

\subsection{Wave height in the gap}

The variation of the non-dimensional averaging wave amplitude $H_{\mathrm{g}} / H_{0}$ in the gap between the twin rolling barges with non-dimensional wave number $k h$ is shown in Fig. 12. In order to demonstrate the influence of the roll motion on the fluid oscillation in the narrow gap, both the numerical simulation and the available experimental data (Saitoh et al., 2006) for the twin fixed barges are also included for comparison. The resonant wave amplitude in the gap between twin rolling barges is almost identical to that between twin fixed barges. The observed resonant wave amplitudes for both numerical cases are approximately $H_{\mathrm{g}} / H_{0}=5.0$, which are the same as the experimental observation. However, the present numerical examination shows that the resonant fre-

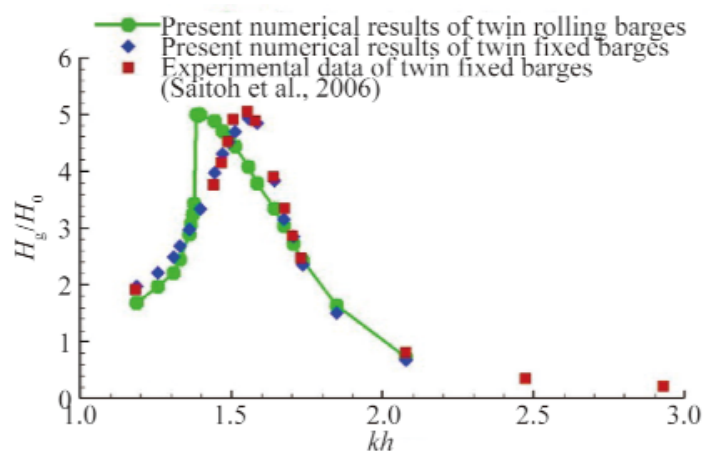

Fig. 12. Wave amplitudes between rolling or fixed barges. 
quency of the fluid oscillation in the narrow gap between the twin rolling barges decreases compared with that between the fixed barges. The resonant frequency for rolling barges is identified at $k h=1.396$ with that at $k h=1.556$ for the twin fixed barges. In addition, much faster increase of the wave amplitude for rolling barges is found as the incident wave frequency approaches the resonant condition, compared with that for fixed barges. The phenomena will be further analyzed in the next section, combined with experimental results of a model test.

\subsection{Roll motion of the twin barges}

Generally, the roll motion of the twin barges is found in the opposite direction. It means if Barge 1 rolls clockwise, Barge 2 must roll anti-clockwise. The wave-induced fluid oscillation in the gap has an opposite effect on the roll motion of the barges. Therefore, the roll directions and equilibrium positions of the twin barges are nearly in the opposite direction. The flow field for the resonant frequency $(k h=1.396)$ in a period is shown in Fig. 13 for an additional illustration. It is obvious that the twin barges roll in the opposite direction. Furthermore, the angular displacements of the twin barges at the incident wave frequency $k h=1.257$, $k h=1.469$ and $k h=1.848$ are also taken for examples in Fig. 14, respectively. The opposite phase of the twin barges is obtained as well. The variation of the roll amplitudes of the twin barges with the incident wave number $k h$ is plotted in Fig. 15, based on the Fourier analysis. Compared with the wave motion in the narrow gap, the overall variation of the roll response is found to be in a similar tendency. The maximum roll amplitudes of the twin rolling barges appear at the same resonant frequency $(k h=1.396)$, which is also the previously identified resonant condition of the fluid oscillation in the narrow gap between the twin rolling barges. By taking the predicted resonant frequency by the potential model into consideration, it is the resonant frequency of the oscillating fluid in the gap that dominates that of the twin rolling barges. In addition, at the lower frequency band the amplitudes of Barge 2 (the rear barge) are larger than those of Barge 1 (the leading barge). Otherwise at the higher frequency band than the resonant frequency, the amplitudes of Barge 1 become larger.

The numerical simulation of this work indicates that the time series of the roll motion of the twin barges are exactly oscillatory with respect to their equilibrium positions, respectively. Fig. 16 shows the equilibrium positions of the twin rolling barges $\zeta_{0}$ in the whole wave numbers considered in this work. The maximum of the mean deflections appears again under the resonant condition $k h=1.396$. It is also shown that the equilibrium positions of the twin barges
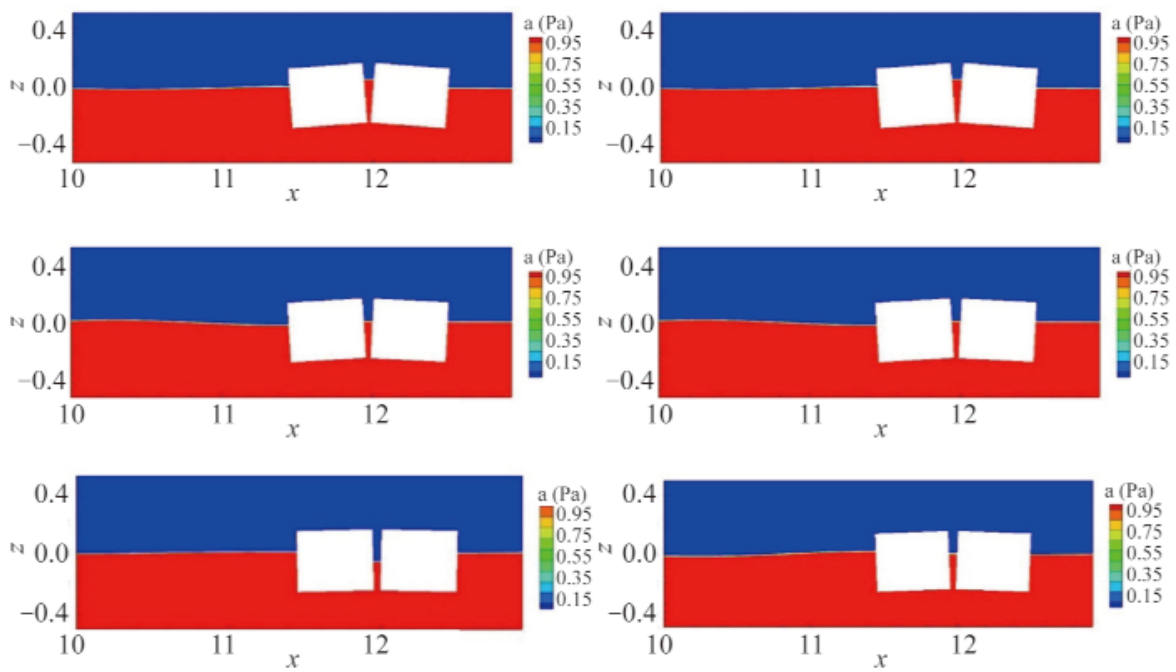

Fig. 13. Flow field in a period for $k h=1.396(T=1.276 \mathrm{~s})$.

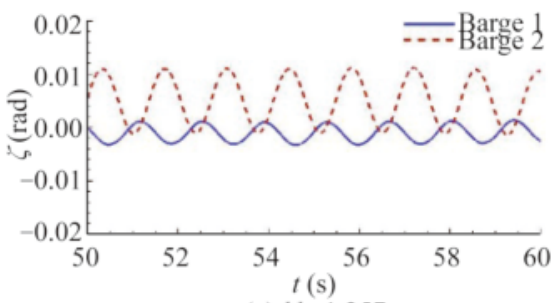

(a) $k h=1.257$

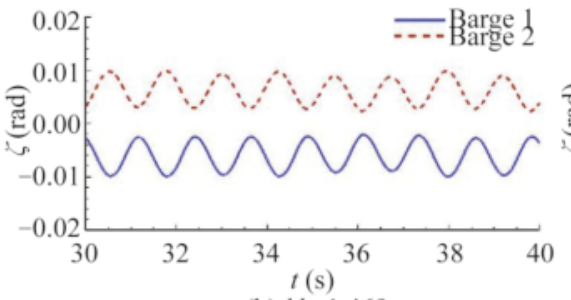

(b) $k h=1.469$

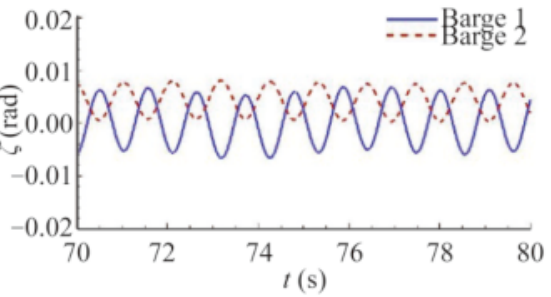

(c) $k h=1.848$

Fig. 14. Time series of the angular displacements for twin barges. 


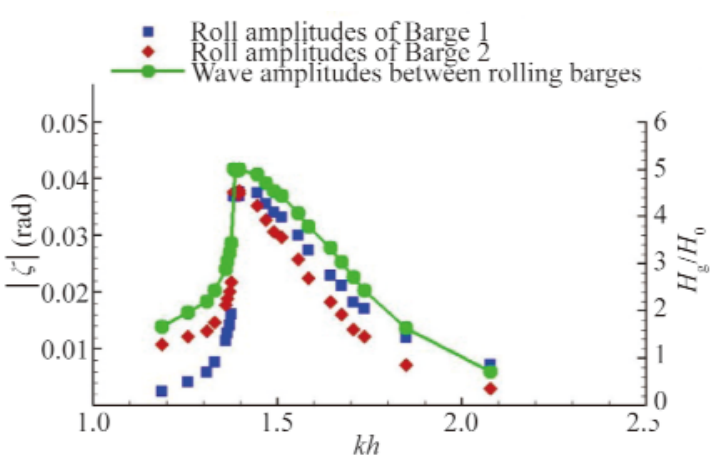

Fig. 15. Roll amplitudes of twin barges.

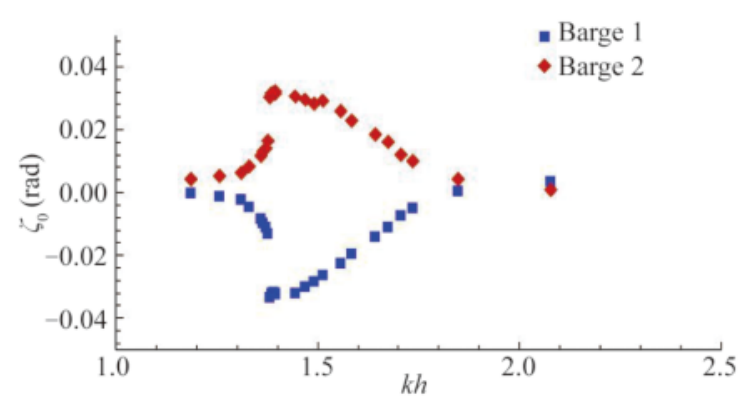

Fig. 16. Equilibrium positions of twin rolling barges.

are antisymmetric with respect to the initial equilibrium positions. It means the Barge 1 presents a mean rotation in an anti-clockwise while the Barge 2 behaves in clockwise. The twin barges are arranged in proximity that the fluid oscillation in the narrow gap is the determinant factor, particularly at the resonant frequency. The fluid oscillation in the gap has an approximate effect on the roll motion of the barges symmetrically, as the twin barges are arranged symmetrically. Therefore, the equilibrium positions of the twin barges are generally identical, but in the opposite direction. Besides, at extremely low and high frequencies, the mean positions approach to the initial vertical direction.

\section{Model experiments of fluid resonance between twin oppositely leaning and fixed side-by-side floating barges}

Based on the aforementioned numerical results in Section 4.4 , it is found that the mean positions of the twin rolling barges deflect oppositely from the initial vertical equilibrium positions. Therefore, a model experiment is carried out to investigate the effects on the fluid resonance due to the deflections of the barge mean positions further. The twin barges are set oppositely leaning and fixed in this experiments. The resonant wave height and frequency with various leaning angles are concerned in this section.

\subsection{Setup of model experiments}

The model experiments were carried out in a wave flume of $69 \mathrm{~m} \times 2.0 \mathrm{~m} \times 1.8 \mathrm{~m}$ of the State Key Laboratory of
Coastal and Offshore Engineering, Dalian University of Technology. An overview of the experimental setup is shown in Fig. 17. A piston wave maker is equipped at the inlet side of the wave flume to generate waves, and a slope beach is arranged at the outlet side of the flume to reduce wave reflection. The wave gauge G5 is located at the narrow gap of twin oppositely leaning and fixed barges to measure the fluid oscillation between them.

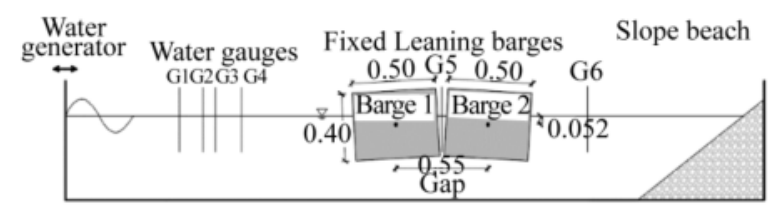

Fig. 17. Sketch of experimental setup for fluid resonance between twin oppositely leaning and fixed side-by-side floating barges.

The model barges are made of plexiglass with a thickness of $1.0 \mathrm{~cm}$ for sufficient rigidity. The size and location of the twin barge are identical to those in the aforementioned numerical simulation, except that the twin barges are set oppositely leaning and fixed. With respect to the rotation center of each barge, the leaning angle $\theta_{\mathrm{L}}$ of each barge is identical but in the opposite direction. Experiments were carried out for five different leaning angles as listed in Table 4 , which are $0^{\circ}$ (vertical), $0.9^{\circ}, 1.4^{\circ}, 2.2^{\circ}$ and $3.3^{\circ}$ relative to the vertical direction, respectively. A picture of the twin leaning model barges with the leaning angle $\theta_{\mathrm{L}}=3.3^{\circ}$ is shown in Fig. 18 for example. The water depth and parameters of the incident waves are also identical to those in the numerical simulation.

Table 4 Parameters of leaning angles

\begin{tabular}{llllll}
\hline Case No. & 1 & 2 & 3 & 4 & 5 \\
\hline$\theta_{\mathrm{L}}\left({ }^{\circ}\right)$ & 0.0 & 0.9 & 1.4 & 2.2 & 3.3 \\
\hline
\end{tabular}

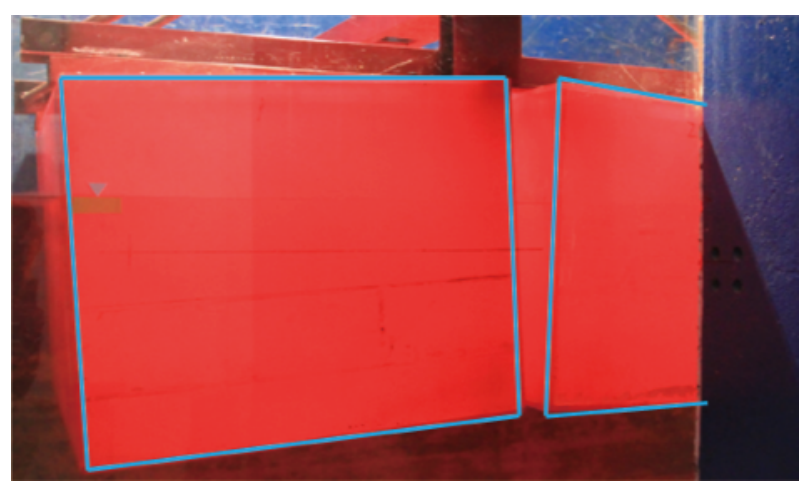

Fig. 18. Twin oppositely leaning and fixed model barges $\left(\theta_{\mathrm{L}}=3.3^{\circ}\right)$.

\subsection{Results and discussions}

In particular, the experimental setup for $\theta_{\mathrm{L}}=0^{\circ}$ degree is identical to that with available experiments (Saitoh et al., 2006), which can be utilized to validate the accuracy of the 
present model experiments. Fig. 19 shows the variation of the non-dimensional averaging wave amplitude $H_{\mathrm{g}} / H_{0}$ in the gap between the twin vertical barges $\left(\theta_{\mathrm{L}}=0^{\circ}\right)$ with non-dimensional wave number $k h$, including the present numerical results. The present experimental results for $\theta_{\mathrm{L}}=0^{\circ}$ agree well with available data, which proves the reliability of the present model experiments.

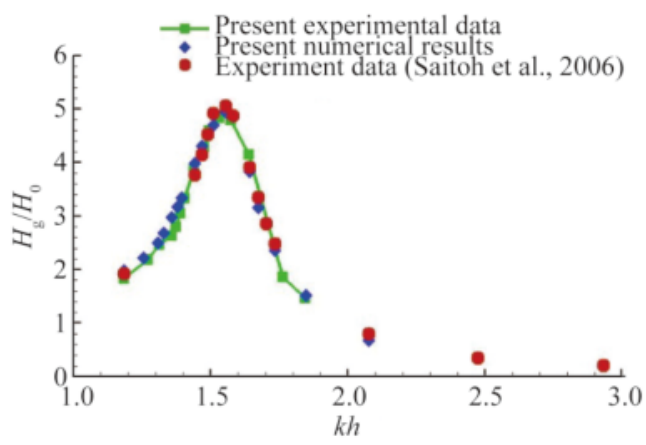

Fig. 19. Wave amplitudes for $\theta_{\mathrm{L}}=0^{\circ}$ (vertical).

The variation of the non-dimensional experimental wave heights in the gap with the non-dimensional wave number $k h$ for various leaning angles is shown in Fig. 20. Both the resonant frequency and resonant wave height of the oscillating fluid in the gap decrease with the increase of the leaning angle. Furthermore, the resonant frequency and the resonant wave height with the leaning angle are illustrated in Figs. 21 and 22, respectively. The potential results of the resonant frequency are also included for comparison in Fig. 21. Generally, the results of the present model experiments agree well with those predicted by the potential mod$\mathrm{el}$, and the resonant frequency has a linear relationship with the leaning angle while the resonant wave height does not. With the increasing lean angle, the mass of the oscillating fluid in the gap increases, which leads to a relatively low resonant frequency. The width of the gap is also broadened from the bottom of the barges to the top with the increasing lean angle. So when resonance happens, an approximate flux at the bottom of the gap leads to a relatively low velocity of the oscillating fluid with the broadening gap width,

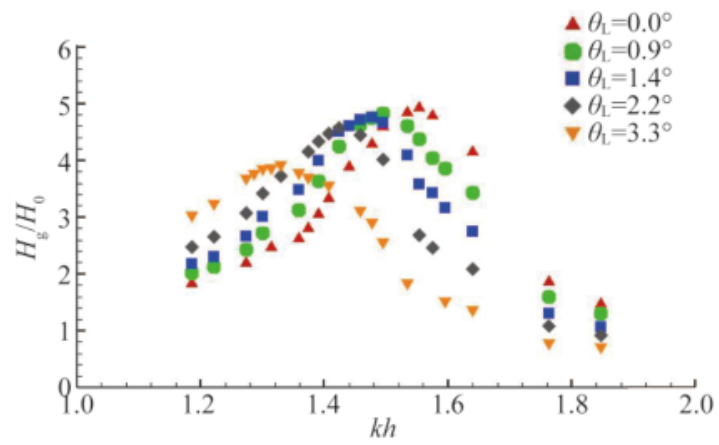

Fig. 20. Wave amplitudes of the present experimental results with leaning angles.

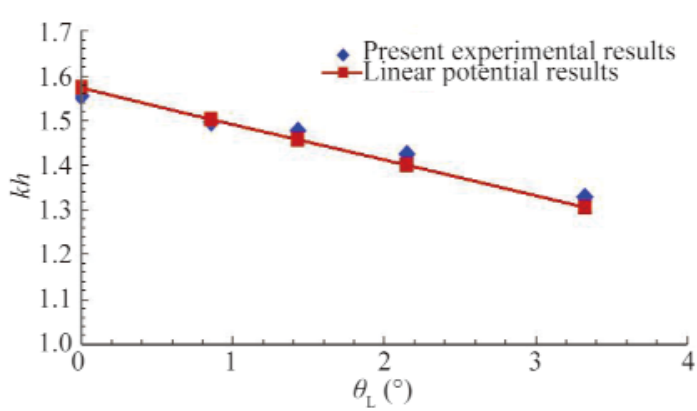

Fig. 21. Resonant frequencies with leaning angles.

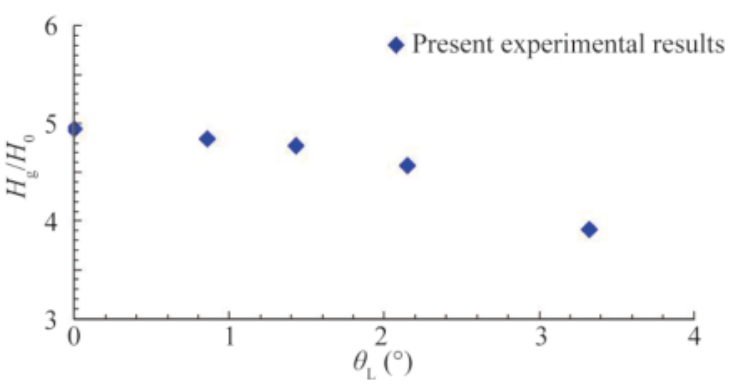

Fig. 22. Resonant wave heights with leaning angles.

which turns out a relatively low resonant wave height in the gap. Besides, the wave height in the narrow gap increases with the increase of the leaning angle within the relatively low frequency band, but decreases with the increase of the leaning angle within the relatively high frequency band.

\subsection{Further analyses}

Based on the aforementioned numerical results of coupling fluid resonance with roll motion of the twin floating barges, the wave height, frequency and deflection of the twin barge equilibrium positions when resonance happens, are $H_{\mathrm{g}} / H_{0}=5.0, k h=1.396(T=1.276 \mathrm{~s})$ and $\left|\zeta_{0}\right|=0.0314 \mathrm{rad}$ $\left(1.8^{\circ}\right)$, respectively. In accordance with the deflection of $1.8^{\circ}$, the corresponding resonant wave height and resonant frequency are $H_{\mathrm{g}} / H_{0}=4.66$, and $k h=1.44(T=1.251 \mathrm{~s})$, interpolated from the experimental results. The resonant frequency predicted by the experimental results is close to that of coupling fluid resonance with roll motion, which indicates that the deflection of the barge equilibrium position is an important factor for the resonant frequency of the coupling motion that mainly matters the mass of the oscillating fluid and flow direction on the barge surface in the gap.

The wave-induced roll motion coupling with gap resonance can be understood as follows. The interaction between the incident wave and the twin rolling barges is focused on in this work. Though the linear wave theory is employed for the incident wave, the numerical model is fully nonlinear. The mean drift torque mainly dominates the deflection of the equilibrium position of the twin rolling barges. Furthermore, a linear relationship between the resonant frequency 
of the fluid in the gap and the deflection of the equilibrium position has been found in the model test, which is shown in Fig. 21. Namely, one deflection matches one resonant frequency of the fluid oscillation in the gap. When the frequency of the incident wave is close to this resonant frequency simultaneously, the fluid resonance in the gap takes place. Besides, the resonant wave amplitude in the gap between twin rolling barges is just obtained from the present numerical simulation. It may be a coincidence that the resonant wave amplitude in the gap between twin rolling barges is identical to that between twin fixed barges.

In summary, two conditions must be fitted in the meantime that the gap resonance takes place. One is the deflection of the equilibrium position due to the wave-induced mean drifted torque, which mainly dominates the resonant frequency, and the other is that the frequency of the incident wave matches the resonant frequency simultaneously. Furthermore, the sharp peak in Fig. 12 can be understood in the following way. Resonance takes place at the "peak", and no resonance at the "foot". As the frequency of the incident wave is close, a faster increase of the wave amplitude for rolling barges is found, compared with that for fixed barges.

\section{Effects of relevant parameters for fluid resonance coupled with roll motion between twin side-by-side floating barges}

Based on the numerical setup in Fig. 10, the influence of relevant parameters for fluid resonance in the gap coupled with roll motion between twin side-by-side barges is examined. The roll moment of inertia of each barge $I=4.305$ $\mathrm{kg} \cdot \mathrm{m}^{2} / \mathrm{m}$, the barge draft $D_{\mathrm{r}}=0.252 \mathrm{~m}$ and the water depth $h=0.5 \mathrm{~m}$ remain as constants, respectively. The rotation center of each barge is invariably located at the corresponding geometry center. The variable parameters are the gap width $B_{\mathrm{g}}$ and the incident wave height $H_{0}$. The wave height in the gap and the roll motion of the twin barges are concerned with various periods of incident waves.

\subsection{Influence of the gap width}

In this section, the incident wave height $H_{0}$ is $0.024 \mathrm{~m}$. The gap width $B_{\mathrm{g}}$ is set as $0.050,0.075$ and $0.10 \mathrm{~m}$, respectively. The variation of the non-dimensional wave amplitude in the gap, the roll amplitudes and the equilibrium positions of the twin barges with non-dimensional wave number $k h$ is shown in Figs. 23-25, respectively. The resonant frequency of the fluid oscillation in the narrow gap and the roll motion of the twin barges keeps identical for each gap width, while it decreases with the increase of the gap width. The resonant wave amplitudes are approximate for the three gap widths, but the phenomena of fast increase of the wave amplitude in the gap fade away with the increasing gap width, when the incident wave frequency approaches the resonant condition. It means that the characteristics of the fast increase of the wave amplitude still exist, but not as

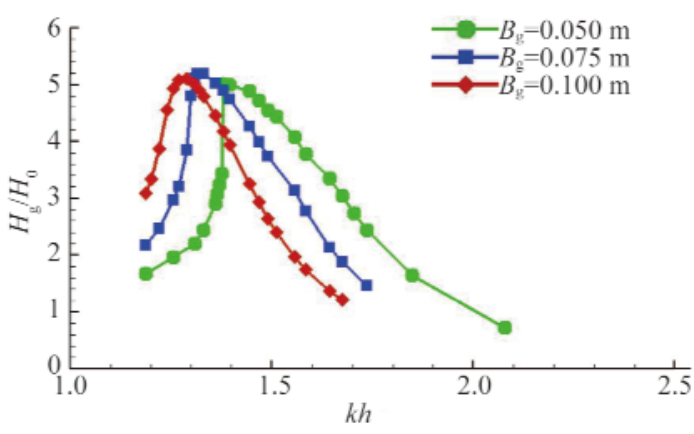

Fig. 23. Wave amplitudes with gap widths.

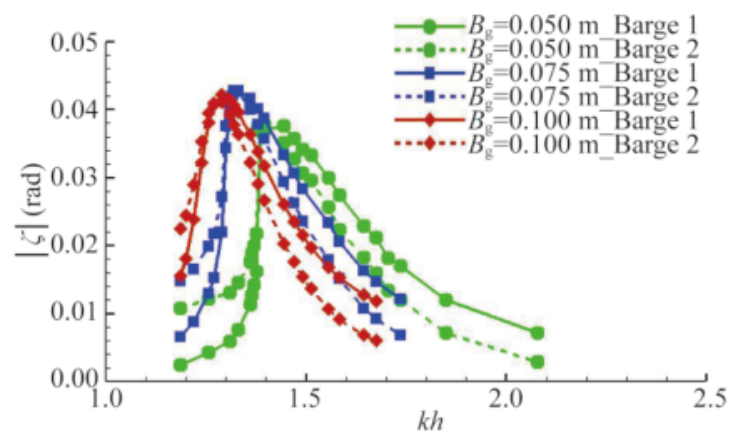

Fig. 24. Roll amplitudes with gap widths.

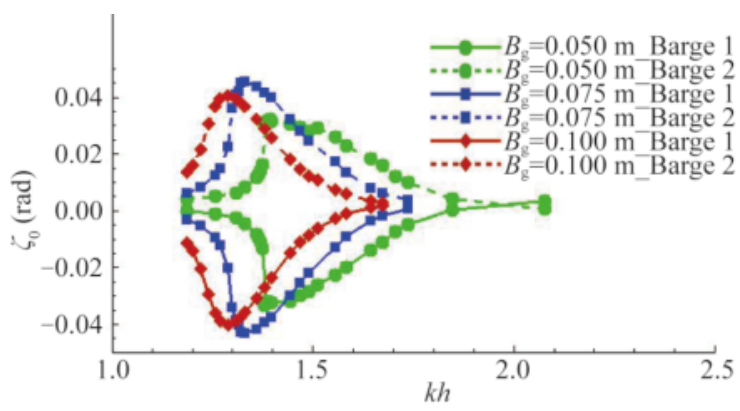

Fig. 25. Equilibrium positions with gap widths.

steep as those for the $0.050 \mathrm{~m}$ gap width. The mass of the oscillating fluid in the gap obviously increases with the broadening gap width, which leads to a relatively low resonant frequency. So the numerical results with different gap widths turn out a similar tendency, while the resonant frequency is shifted. In addition, the roll amplitudes of Barge 1 and Barge 2 become larger with the wider gap within the lower frequency band, while lower within the higher frequency band. The similarity for the equilibrium positions of the twin barges with various gap widths is found as well.

\subsection{Influence of the incident wave height}

In this section, the gap width $B_{\mathrm{g}}$ is $0.050 \mathrm{~m}$. The incident wave height $H_{0}$ is set as $0.012,0.024$ and $0.030 \mathrm{~m}$, respectively. The variation of the non-dimensional wave amplitude in the gap, the roll amplitudes and the equilibrium po- 
sitions of the twin barges with non-dimensional wave number $k h$ are shown in Figs. 26-28, respectively. The resonant frequency of the fluid oscillation in the narrow gap and the roll motion of the twin barges keeps identical for each incident wave height, while it decreases with the increase of the incident wave height. The non-dimensional wave amplitude in the gap is lower for the incident wave with higher wave height. The roll amplitudes of Barge 1 and Barge 2 are larger with the higher incident wave height, so are the deflections of their equilibrium positions. As mentioned in Section 5.3, the mean drift torque mainly dominates the deflection of the equilibrium position of the twin rolling barges. One deflection matches one resonant frequency of the fluid oscillation in the gap. Furthermore, it is a direct ratio relation between the mean drift torque and the square of the in-

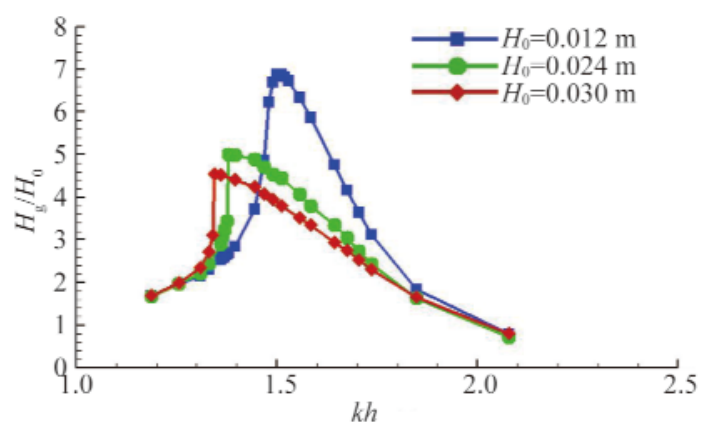

Fig. 26. Wave amplitudes with incident wave heights.

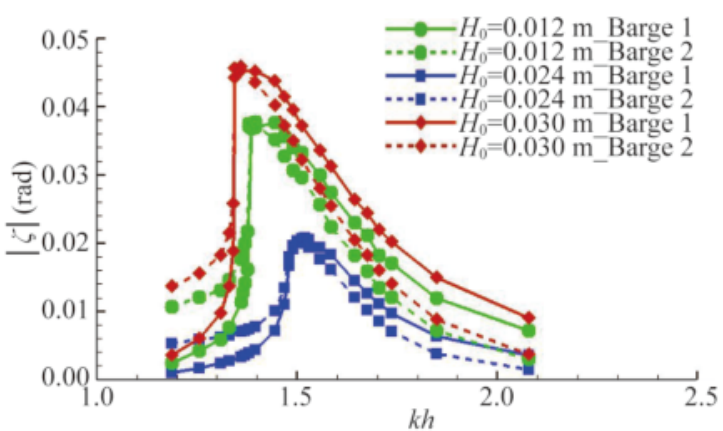

Fig. 27. Roll amplitudes with incident wave heights.

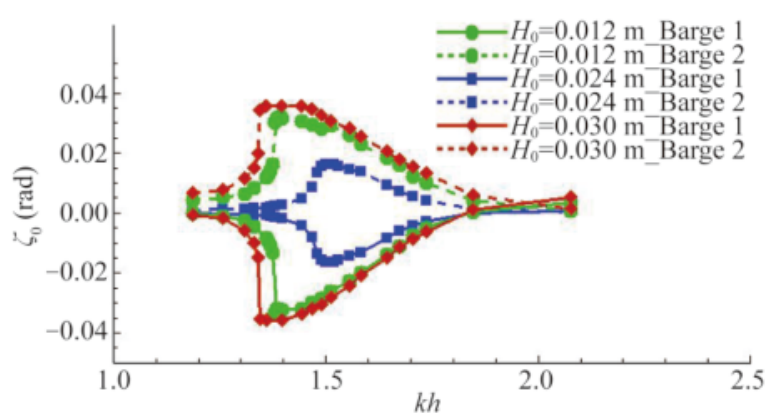

Fig. 28. Equilibrium positions with incident wave heights. cident wave height. When the incident wave height is different, the deflection also varies. So the resonant frequency turns out different. For the decreasing non-dimensional resonant wave amplitude in the gap with the increasing incident wave height, it owes to the effect of the nonlinearity.

\section{Conclusions}

The open source CFD package, OpenFOAM, is developed to study the coupling effects of fluid resonance and roll motion between twin rectangular barges in proximity, subjected to incident waves normal to the spanwise of the narrow gap in a 2D numerical wave flume. After numerical validations are achieved, the present numerical model is utilized to investigate the resonant wave height and the resonant frequency in the narrow gap between the twin rolling barges, besides the characteristics of their roll motion. Based on the present numerical results, a model experiment is conducted for a further investigation of the resonant wave height and the resonant frequency in the narrow gap between twin oppositely leaning and fixed barges. In addition, the effects of the gap width and incident wave height on the fluid resonance coupled with roll motion between the twin barges are examined. Through this work, the following conclusions can be drawn.

(1) The present numerical results illustrate that the resonant frequency of the oscillating fluid coupled with roll motion of the twin barges is determined by the frequency of the gap resonance. The resonant frequency is lower than that between twin fixed barges. The roll displacements of the twin barges are generally in the opposite direction. Besides, the equilibrium position of the roll motion for the leading barge deflects in the anti-clockwise respect to the initial vertical direction, while for the rear barge in the clockwise simultaneously.

(2) From the present experimental results of the fluid resonance in the gap between twin oppositely leaning and fixed barges, it is found that both the resonant frequency and wave height in the gap decrease with the increase of the leaning angle, while the resonant frequency turns out in a linear way. Furthermore, the deflection of the equilibrium position of the twin barges is a relevant factor for the decrease of the resonant frequency between the twin rolling barges, compared with that between the twin fixed barges. The deflection mainly matters the mass of the oscillating fluid and flow direction on the barge surface in the gap.

(3) For the fluid resonance coupled with roll motion between twin side-by-side barges, the effects of the gap width and incident wave height are as follows. The increase of the gap width decreases the resonant frequency, while the resonant wave height in the gap is nearly unaffected. However, both the resonant frequency and the resonant wave height in the gap decrease with the increase of the incident wave height. 


\section{Right and permissions}

Open Access This article is licensed under a Creative Commons Attribution 4.0 International License, which permits use, sharing, adaptation, distribution and reproduction in any medium or format, as long as you give appropriate credit to the original author(s) and the source, provide a link to the Creative Commons licence, and indicate if changes were made. The images or other third party material in this article are included in the article's Creative Commons licence, unless indicated otherwise in a credit line to the material. If material is not included in the article's Creative Commons licence and your intended use is not permitted by statutory regulation or exceeds the permitted use, you will need to obtain permission directly from the copyright holder. To view a copy of this licence, visit http://creativecommons.org/licenses $/$ by $/ 4.0 /$.

\section{References}

Calderer, A., Kang, S. and Sotiropoulos, F., 2014. Level set immersed boundary method for coupled simulation of air/water interaction with complex floating structures, Journal of Computational Physics, 277, 201-227.

Choi, Y.R. and Hong, S.Y., 2002. An analysis of hydrodynamic interaction of floating multi-body using higher-order boundary element method, Proceedings of the 12th International Offshore and Polar Engineering Conference, Kitakyushu, Japan.

Hirt, C.W., Amsden, A.A. and Cook, J.L., 1997. An arbitrary Lagrangian-Eulerian computing method for all flow speeds, Journal of Computational Physics, 135(2), 203-216.

Hirt, C.W. and Nichols, B.D., 1981. Volume of fluid (VOF) method for the dynamics of free boundaries, Journal of Computational Physics, 39(1), 201-225.

Hong, S.Y., Kim, J.H., Cho, S.K., Choi, Y.R. and Kim, Y.S., 2005. Numerical and experimental study on hydrodynamic interaction of side-by-side moored multiple vessels, Ocean Engineering, 32(7), 783-801.

Iwata, H., Saitoh, T. and Miao, G.P., 2007. Fluid resonance in narrow gaps of very large floating structure composed of rectangular modules, Proceedings of the 4th International Conference on Asian and Pacific Coats, Nanjing, China.

Jasak, H. and Tukovic, Z., 2007. Automatic mesh motion for the unstructured finite volume method, Transactions of FAMENA, 30(2), $1-20$.

Jiang, S.C., Bai, W. and Tang, G.Q., 2018. Numerical simulation of wave resonance in the narrow gap between two non-identical boxes, Ocean Engineering, 156, 38-60.

Jin, R.J., Teng, B., Ning, D.Z., Zhao, M. and Cheng, L., 2017. Numerical investigation of influence of wave directionality on the water resonance at a narrow gap between two rectangular barges, Acta Oceanologica Sinica, 36(6), 104-111.

Jung, K.H., Chang, K.A. and Jo, H.J., 2006. Viscous effect on the roll motion of a rectangular structure, Journal of Engineering Mechanics, 132(2), 190-200.

Kashiwagi, M., Endo, K. and Yamaguchi, H., 2005. Wave drift forces and moments on two ships arranged side by side in waves, Ocean Engineering, 32(5-6), 529-555.

Kristiansen, T. and Faltinsen, O.M., 2010. A two-dimensional numerical and experimental study of resonant coupled ship and piston- mode motion, Applied Ocean Research, 32(2), 158-176.

Larsen, J. and Dancy, H., 1983. Open boundaries in short wave simulations-a new approach, Coastal Engineering, 7(3), 285-297.

Li, B.N., Cheng, L., Deeks A.J. and Teng, B., 2005. A modified scaled boundary finite-element method for problems with parallel sidefaces. Part II. Application and evaluation, Applied Ocean Research, 27(4-5), 224-234.

Lu, L., Chen, X.B. and Teng, B., 2012. Viscous dissipation and artificial damping for gap resonance problem, Proceedings of the 10th International Conference on Hydrodynamics, Saint Petersburg, Russia.

Lu, L., Cheng, L., Teng, B. and Li, Y.C., 2008. Numerical simulation of hydrodynamic resonance in a narrow gap between twin bodies subject to water waves, Proceedings of the 18th International Offshore and Polar Engineering Conference, Vancouver, Canada.

Lu, L., Cheng, L., Teng, B. and Zhao, M., 2010. Numerical investigation of fluid resonance in two narrow gaps of three identical rectangular structures, Applied Ocean Research, 32(2), 177-190.

McIver, P., 2005. Complex resonances in the water-wave problem for a floating structure, Journal of Fluid Mechanics, 536, 423-443.

McIver, P. and Porter, R., 2016. The motion of a freely floating cylinder in the presence of a wall and the approximation of resonances, Journal of Fluid Mechanics, 795, 581-610.

Meringolo, D.D., Liu, Y. and Lu, L., 2018. Energy analysis of wave resonance in a gap through an SPH model, Proceedings of the 28th International Ocean and Polar Engineering Conference, Sapporo, Japan.

Miao, G.P., Saitoh, T. and Ishida, H., 2001. Water wave interaction of twin large scale caissons with a small gap between, Coastal Engineering Journal, 43(1), 39-58.

Ning, D.Z., Zhu, Y., Zhang, C.W. and Zhao, M., 2018. Experimental and numerical study on wave response at the gap between two barges of different draughts, Applied Ocean Research, 77, 14-25.

Nwogu, O., 1993. Alternative form of Boussinesq equations for nearshore wave propagation, Journal of Waterway, Port, Coastal, and Ocean Engineering, 119(6), 618-638.

Romate, J.E., 1992. Absorbing boundary conditions for free surface waves, Journal of Computational Physics, 99(1), 135-145.

Rusche, H., 2002. Computational Fluid Dynamics of Dispersed TwoPhase Flows at High Phase Fractions. Ph.D. thesis, Imperial College, London.

Saitoh, T., Miao, G.P. and Ishida, H., 2006. Theoretical analysis on appearance condition of fluid resonance in a narrow gap between two modules of very large floating structure, Proceedings of the $3 \mathrm{rd}$ Asia-Pacific Workshop on Marine Hydrodynamics, Shanghai, China.

Sun, L., Taylor, R.E. and Taylor, P.H., 2010. First- and second-order analysis of resonant waves between adjacent barges, Journal of Fluids and Structures, 26(6), 954-978.

Sun, L., Taylor, R.E. and Taylor, P.H., 2015. Wave driven free surface motion in the gap between a tanker and an FLNG barge, Applied Ocean Research, 51, 331-349.

Tan, L., Lu, L., Tang, G.Q. and Cheng, L., 2016. Experimental study of wave resonance in a narrow gap with various edge shapes, Proceedings of the 20th Australasian Fluid Mechanics Conference, Perth, Australia.

Tan, L., Lu, L., Tang, G.Q., Cheng, L. and Chen, X.B., 2019. A viscous damping model for piston mode resonance, Journal of Fluid Mechanics, 871, 510-533.

Teng, B. and Taylor, R.E., 1995. New higher-order boundary element 
methods for wave diffraction/radiation, Applied Ocean Research, 17(2), 71-77.

Ubbink, O. and Issa, R.I., 1999. A method for capturing sharp fluid interfaces on arbitrary meshes, Journal of Computational Physics, 153(1), 26-50.

Wang, D.G. and Zou, Z.L., 2007. Study of non-linear wave motions and wave forces on ship sections against vertical quay in a harbor, Ocean Engineering, 34(8-9), 1245-1256.

Wei, G., Kirby, J.T. and Sinha, A., 1999. Generation of waves in
Boussinesq models using a source function method, Coastal Engineering, 36(4), 271-299.

Yan, S.Q., Ma, Q.W. and Cheng, X.M., 2009. Fully nonlinear hydrodynamic interaction between two 3D floating structures in close proximity, Proceedings of the 19th International Offshore and Polar Engineering Conference, Osaka, Japan.

Zhang, J.W., He, G.H., Mei, L. and Zhang, D.H., 2018. Gap influence on wave interaction of twin caissons in close proximity, International Journal of Engineering and Technology, 10(1), 18-22. 